\title{
Outcomes of patients lost to followup after mid urethral synthetic slings--successes or failures?
}

\author{
Ballert KN, Rose AE, Biggs GY, Rosenblum N, Nitti VW
}

Division of Urology, University of Kentucky, Lexington, Kentucky, USA

J Urol. 2010; 183: 1455-8

Purpose: We classified patients lost to follow-up after mid urethral synthetic sling placement as examples of treatment success or failure based on the Patient Global Impression of Improvement, and compared the outcomes of those who followed up to the outcomes of those who did not.

Materials and Methods: We reviewed the charts of 217 patients who underwent mid urethral synthetic sling placement. Telephone interviews including the Patient Global Impression of Improvement and the Medical, Epidemiological, and Social Aspects of Aging questionnaires were conducted for patients lacking 3-month follow-up.

Results: Based on the Patient Global Impression of Improvement of the 48 patients who responded 13 (27.1\%) were failures. The overall failure rate of patients with at least 3-month follow-up was 19\% (23 of 124).

Conclusions: In our study success rates for patients lost to follow-up were similar to the rates for those who had routine follow-up. However, it is uncertain if these data can be applied to other study populations, especially in a randomized controlled trial.

\section{Editorial Comment}

Notable experts in the field address the question of "what is the status of women who were lost to follow-up after their mid urethral sling surgery?". They classified "lost to follow-up" as patients who did not return for their 3 months post operative appointments. Those patients lost to follow up, when identified and contacted, appeared to have very similar results to those patients who were able to be followed. The authors note the utility of the PGI-I one item questionnaire when assessing patients post-operatively after therapy for stress urinary incontinence. The paper did note that even when trying to contact all the patients lost to follow-up, there still remained a small population of patients who could not be contacted thus were truly lost to follow-up. It seems that not all lost to follow-up patients are failures or successes; even with including that truly lost to follow-up population, those patients who did not report for their postoperative follow-up have very similar outcomes to those who did thus potentially aiding analysis of future post sling reports.

Dr. Steven P. Petrou

Professor of Urology, Associate Dean Mayo School of Graduate Medical Education

Jacksonville, Florida, USA

E-mail:petrou.steven@mayo.edu 